\title{
nature
}

\section{Joys of darkness}

Planetaria are getting ever better at capturing the glories and the challenges of the night sky. They are underused not only in that astronomical context, but for other sciences too.

$\mathrm{O}$ $\mathrm{f}$ all people, New Yorkers face the biggest obstacles to experiencing the magic of a moonless, starry night. The absolute quiet, the spangled black panorama, the misty billions of stars in the Milky Way, the sense of majestic timelessness. No wonder, having lost all this, New Yorkers top the league tables for rancour. But they have been partially compensated: since February, they have been able to enjoy the Hayden Planetarium at the American Museum of Natural History.

For this technological achievement and for the great spectacle it provides, all credit to the engineers of the Carl Zeiss planetarium division in Jena, eastern Germany; to the Hubble Space Telescope, which is the source of many of the planetarium's best images; and to countless developments over the past decade or two in the understanding of the structure and content of our stellar neighbourhood, of our Galaxy as a whole, and of the largest scales of the detectable Universe.

The Hayden's technology is worth highlighting. The "Universarium" projector, like conventional planetarium projectors, uses an arc lamp to give appropriate colour balances and star masks by which the stellar images are projected onto the dome. One innovation is to use fibre optics to concentrate the light onto the holes in the masks and thereby allow a hundredfold increase in brilliance. Another is to draw on stellar position data from an unprecedentedly large database compiled by the US space agency NASA, allowing stars to be projected that are below most people's thresholds of visible detection. (Visitors can bring binoculars to see for themselves.) Then there are the more conventional facilities - separate projectors for more mobile sky objects such as the planets and the Moon, multiple projectors for conventional images spanning the dome's surface, and computersimulation projection.

\section{Fulfilment and frustration}

The half-hour show Passport to the Universe is routinely sold out at the Hayden several times a day. Anyone with any knowledge of astronomy - even an amateur acquaintance with the night sky - is certain to find it fulfilling and frustrating. Fulfilling not only because of the sheer quality of the images, but also because nowhere else will they have seen such a fine and up-to-date visualization of the distribution of superclusters of galaxies that makes up the largest-scale structure of the Universe, or such a superb rendering of an imaginary trip through the Orion nebula, a 'nearby' gas cloud in which stars are being formed. Frustrating, however, because these are merely glimpsed and yet contain glories and mysteries enough in themselves to sustain more extensive display - the dynamics of motions of groups of galaxies, for example, and the evolution of the Universe's structure. Included in the show's portrait of the Orion nebula, but unremarked, is a protostar complete with accreting gas and jets of ejected material - another fine feature to focus on, with a possible speculative extension into the growing numbers of stars around which have been detected preplanetary clouds, protoplanetary disks, brown dwarfs and planets.

Such phenomena are at the forefront of current research. But it would be regrettable if, as the imaging improves, newly discovered phenomena crowded out the traditional portrayal and explanation of well-known features of our stellar neighbourhood: long-understood objects visible as mere dots with the naked eye, such as the zoo of variable stars, both single (such as $\gamma$ Cassiopeia, a white-hot star that discards material in occasional bursts) and multiple (including distorted interacting stars as they orbit their common centre of mass); and the variety of beautiful star clusters, 'open' (such as the Pleiades) and globular ( $\omega$ Centauri).

\section{Opportunities}

Given the popularity of the Hayden shows, it is clear that there is an appetite for far more to be shown. And with at least 1,500 planetaria worldwide, albeit less sophisticated than that in New York, it is also clear that the opportunities for better exploitation of such facilities are global and growing.

Many planetaria are to be found at science centres, yet seem all too often to be considered as purely astronomical show-places. Much of the technical effort is devoted to precise reproduction of stellar and planetary motions, and to perspectives from different Galactic locations and eras. But planetaria possess more conventional projection facilities that will surely blossom if allied with two other technologies: supercomputing simulation and computer-based animation. Might most planetaria become 'scientaria'?

Visit any supercomputing facility and you will probably encounter simulations not only of astronomical processes but also of molecular interactions, electronic materials and complex fluid flows. Enclose the observer in a planetarium hemisphere and project these with due imagination, and you could quite quickly compel their attention to the complex beauty of the processes at work, thereby, incidentally, unlocking some of the black boxes of everyday technology. Turn to the animators, and imaginary but scientifically insightful voyages through cells become a possibility. Given very recent progress, the structure and function of key cell constituents such as ribosomes and membrane channels can now be speculatively but credibly visualized. What better way to put into biological context the progress of the Human Genome Project than by portraying the chain of action from genome to individual gene expressions and their macroscopic outcomes, and how these are influenced by environmental signals?

Whether the current planetarium suppliers - Zeiss, Spitz and the like - or the growing numbers of more conventional 'immersive theatre' companies can profitably fulfil this opportunity remains to be seen. But millions of people every year would benefit from the results. And of course, all of this is but a step towards virtual reality, in which any observer can happily immerse themselves in his or her own simulation or database, and which supercomputer centres are already exploiting.

Immersive visualization is not the same as understanding, but, as New Yorkers might well agree, it's a great step along the way, and far closer to the magic of the real world, night and day, than the Discovery channel. 